\title{
Protein-Embedded Metalloporphyrin Arrays Templated by Circularly Permuted Tobacco Mosaic Virus Coat Proteins
}

Jing Dai, ${ }^{a}$ Gavin J. Knott, ${ }^{\mathrm{b}}$ Wen Fu, ${ }^{\mathrm{c}}$ Tiffany W. Lin, ${ }^{\mathrm{a}, \dagger}$ Ariel L. Furst, ${ }^{\mathrm{d}}$ R. David Britt, ${ }^{\mathrm{c}}$ and Matthew B. Francis, ${ }^{\mathrm{a}, \mathrm{e},}$,

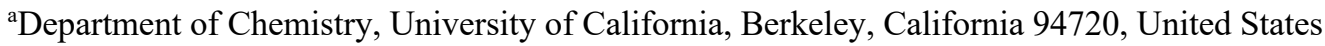

${ }^{b}$ Department of Molecular and Cell Biology, University of California, Berkeley, California 94720, United States

${ }^{\mathrm{c}}$ Department of Chemistry, University of California, One Shields Avenue, Davis, California 95616, United States

${ }^{\mathrm{d} D e p a r t m e n t}$ of Chemical Engineering, Massachusetts Institute of Technology, Cambridge, Massachusetts 02139, United States

${ }^{\mathrm{e}}$ Materials Sciences Division and Molecular Biophysics and Integrated Bioimaging Division, Lawrence Berkeley National Laboratories, Berkeley, California 94720, United States

†Late Stage Pharmaceutical Development, Genentech Inc., 1 DNA Way, South San Francisco, California 94080, United States

*Correspondence should be addressed to mbfrancis@berkeley.edu

\section{SUPPORTING INFORMATION}

\section{Table of Contents}

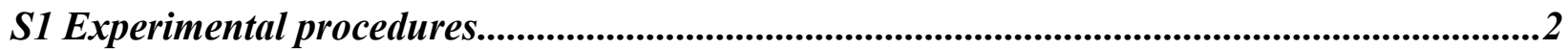

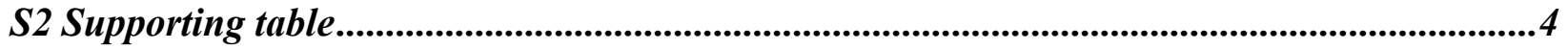

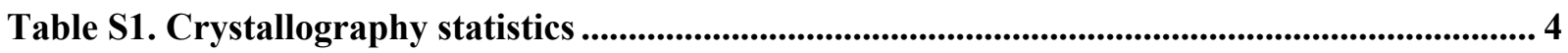

S3 Supporting figures ...............................................................................................................................5

Figure S1. Constraints for computational minimization ..................................................................5

Figure S2. Computationally modeled heme binding pockets of six epTMV mutants........................ 6

Figure S3. Characterization of cpTMV mutants assembly states ........................................................ 7

Figure S4. Characterization of heme binding events of cpTMV mutants .......................................... 8

Figure S5. Structural comparisons for three cpTMV structures ............................................................9

Figure S6. Analysis of the protein-protein interaction changes upon heme binding....................... 10

Figure S7. Characterization of cpTMV-Q101H binding to other types of metal porphyrins........ 11

Figure S8. Modeled interaction between the heme and protein side chains.................................. 12

Figure S9. Temperature dependent EPR spectra ................................................................................ 12

Figure S10. Arrangement of heme arrays templated by cpTMV ...................................................... 13 


\section{S1 Experimental procedures}

General methods and materials. Unless otherwise noted, all chemicals and solvents were of analytical grade and were received from commercial sources. All the metalloporphyrins were purchased from Frontier Scientific Inc. (Logan, UT). Water $\left(\mathrm{dd}-\mathrm{H}_{2} \mathrm{O}\right)$ used as reaction solvent was deionized using a Barnstead NANOpure purification system (ThermoFisher, Waltham, MA). All oligonucleotides were purchased from Integrated DNA Technologies (Coralville, IA). Spin concentration was performed using 100,000 molecular weight cutoff spin concentrators from Millipore (Burlington, MA). Dialysis was performed with either Slide-A-Lyzer Dialysis Cassettes (Pierce, Rockford, Il) for small volume samples or dialysis tubing (Fisherbrand, Pittsburgh, PA) for large volume samples. All the experiments were done under aerobic conditions and the hemes stayed in their oxidized forms ( $\mathrm{Fe}(\mathrm{III})$ protoporphyrin) unless specifically mentioned otherwise.

Generation of cpTMV mutants. The gene for the permuted TMV coat protein (cpTMVP) was cloned into an E. coli expression vector (pET 20b, Novagen) following the protocol reported earlier. ${ }^{3}$ The QuickChange II Site-Directed Mutagenesis Kit (Agilent Technologies, USA) was used to generate cpTMV-Q101H, cpTMV-V105H, cpTMV-R108H, cpTMV-Q109H, cpTMVE112H and cpTMV-P112H plasmids from the cpTMV-nohis plasmid using the following sets of primers:

Q101H:

Sense: 5' GGCAACCAATTTCAGACCCAGCATGCGCGCACCG 3' Antisense: 5' GCACAACGGTGCGCGCATGCTGGGTCTGAAATTGG 3'

V105H:

Sense: 5' GCAGGCGCGCACCCATGTGCAGCGTCAGTTCAGC 3'

Antisense: 5' GCTGAACTGACGCTGCACATGGGTGCGCGCCTGC 3'

R108H:

Sense: 5' GGCGCGCACCGTTGTGCAGCATCAGTTCAGCGAAGTTTGG 3' Antisense: 5' GGTTTCCAAACTTCGCTGAACTGATGCTGCACAACGGTGC 3' Q109H:

Sense: 5' GCGCACCGTTGTGCAGCGTCATTTCAGCGAAGTTTGGAAACC 3' Antisense:5' GGCTCGGTTTCCAAACTTCGCTGAAATGACGCTGCACAACGG 3' E112H:

Sense: 5' CGTTGTGCAGCGTCAGTTCAGCCATGTTTGGAAACCGAGC 3' Antisense: 5' GCGGGCTCGGTTTCCAAACATGGCTGAACTGACGCTGC 3' P116H:

Sense: 5' GCAGCGTCAGTTCAGCGAAGTTTGGAAACATAGCCCGCAGG 3' Antisense: 5' GGTAACCTGCGGGCTATGTTTCCAAACTTCGCTGAACTGACG 3' 
Mass spectrometry. Protein and small molecules were analyzed using an Agilent 1200 series liquid chromatograph (Agilent Technologies, USA) that was connected in-line with an Agilent 6224 Time-of-Flight (TOF) LC/MS system equipped with a Turbospray ion source. Protein samples were run with a Proswift RP-4H column (Dionex, USA). Protein mass reconstruction was performed on the charge ladder with Mass Hunter software (Agilent, USA).

High Performance Liquid Chromatography. HPLC was performed on Agilent 1260 Infinity Series HPLC Systems (Agilent, USA). Sample analysis for all HPLC experiments was achieved with an in-line diode array detector (DAD) and in-line fluorescence detector (FLD). Size exclusion chromatography (SEC) was performed using a Polysep-GFC-P-5000 column (4.6 x $250 \mathrm{~mm})$ (Phenomenex, USA) at $1.0 \mathrm{~mL} / \mathrm{min}$ using a mobile phase of $10 \mathrm{mM}$ sodium phosphate buffer, $\mathrm{pH}$ 7.2 .

Gel Analysis. Sodium dodecyl sulfate-polyacrylamide gel electrophoresis (SDS-PAGE) was carried out in a Mini cell tank apparatus (Life Technologies, Carlsbad, CA), using NuPAGE ${ }^{\text {TM }}$ Novex ${ }^{\mathrm{TM}} 4-12 \%$ Bis-Tris Protein Gels (Life Technologies). The sample and electrode buffers were prepared according to the suggestions of the manufacturer. All protein electrophoresis samples were heated for $5-10 \mathrm{~min}$ at $95^{\circ} \mathrm{C}$ in the presence of 1,4-dithiothreitol (DTT) to ensure reduction of disulfide bonds. Gels were run for $40 \mathrm{~min}$ at $200 \mathrm{~V}$ to separate the bands. Commercially available markers (Bio-Rad) were applied to at least one lane of each gel for assignment of apparent molecular masses. Visualization of protein bands was accomplished by staining with Coomassie Brilliant Blue R-250 (Bio-Rad, Hercules, CA). Gel imaging was performed on a Gel Doc (Bio-Rad, Hercules, CA). 
S2 Supporting table

\begin{tabular}{|c|c|c|}
\hline & $\begin{array}{l}\text { cpTMV Q101H apoprotein } \\
\text { (PDB code: 6X0R) }\end{array}$ & $\begin{array}{l}\text { cpTMV Q101H holoprotein } \\
\text { (PDB code: 6X0Q) }\end{array}$ \\
\hline \multicolumn{3}{|l|}{ Data collection } \\
\hline Space group & $C 121$ & $C 121$ \\
\hline \multicolumn{3}{|l|}{ Cell dimensions } \\
\hline$a, b, c(\AA)$ & $218.70,176.21,102.25$ & $218.01,176.14,100.24$ \\
\hline$\alpha \beta \gamma\left(^{\circ}\right)$ & $90.00,99.16,90.00$ & $90.00,98.53,90.00$ \\
\hline Wavelength $(\AA)$ & 1.1158 & 1.1158 \\
\hline Resolution $(\AA)$ & $136.52-2.81(2.86-2.81)$ & $136.40-3.00(3.06-3.00)$ \\
\hline$R_{\text {merge }}$ & $0.111(1.544)$ & $0.671(5.496)$ \\
\hline$<I / \sigma(I)>$ & $9.8(1.1)$ & $4.5(0.5)$ \\
\hline$C C_{1 / 2}$ & $0.999(0.651)$ & $0.983(0.317)$ \\
\hline Completeness (\%) & $98.0(88.2)$ & $100.0(100.0)$ \\
\hline Redundancy & $6.9(6.0)$ & $12.3(12.3)$ \\
\hline \multicolumn{3}{|l|}{ Refinement } \\
\hline Resolution ( $\AA$ ) & $48.97-3.00(3.07-3.00)$ & $49.57-3.00(3.07-3.00)$ \\
\hline No. reflections & 73236 & 74481 \\
\hline$R_{\text {work }} / R_{\text {free }}$ & $0.269 / 0.289$ & $0.257 / 0.265$ \\
\hline No. atoms & 18581 & 18,985 \\
\hline Protein & 18581 & 18598 \\
\hline Heme & - & 387 \\
\hline \multicolumn{3}{|l|}{$B$ factors } \\
\hline Protein & 126.0 & 58.58 \\
\hline Ligand/ion & - & 67.70 \\
\hline \multicolumn{3}{|l|}{ R.m.s. deviations } \\
\hline Bond lengths $(\AA)$ & 0.002 & 0.002 \\
\hline Bond angles $\left({ }^{\circ}\right)$ & 0.451 & 0.501 \\
\hline
\end{tabular}

Table S1. Crystallography statistics. 


\section{S3 Supporting figures}

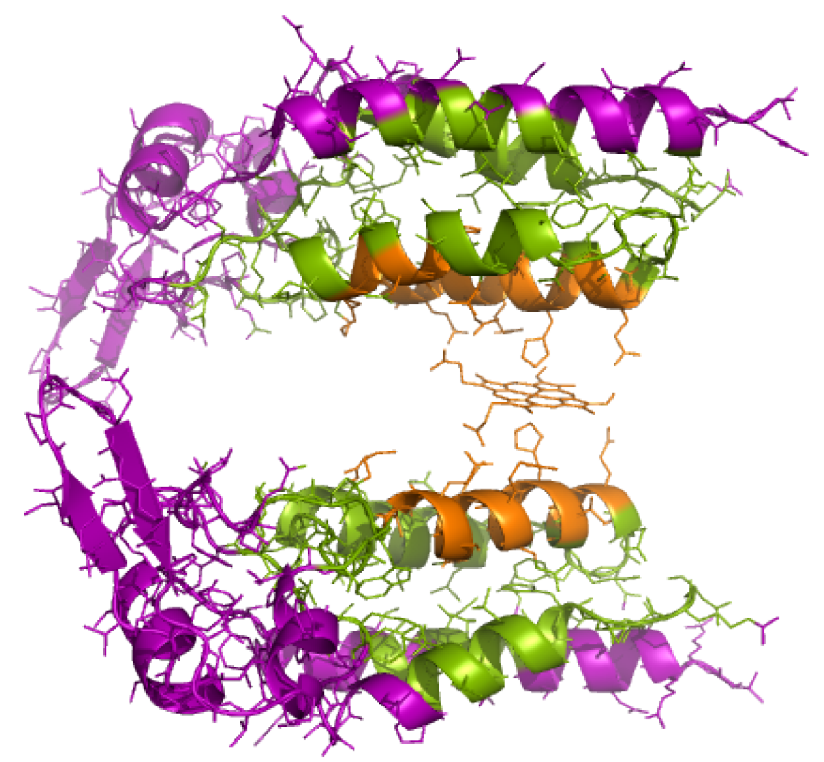

Figure S1. Constraints for computational minimization.Three layers were set with different freedom constraints in computational modeling to save computational time. The shell within $10 \AA$ of the histidine mutation sites, colored orange, was set to move freely. The residues within $20 \AA$ but beyond $10 \AA$, colored green, were constrained. The shell beyond $20 \AA$, colored purple, was frozen. 
a
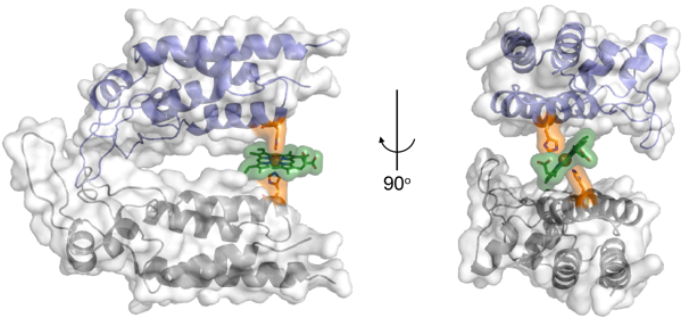

c

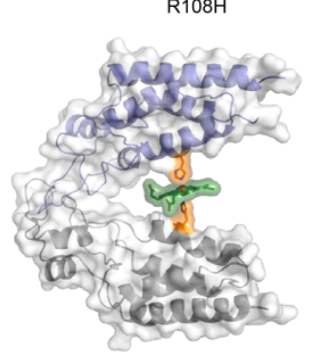

e

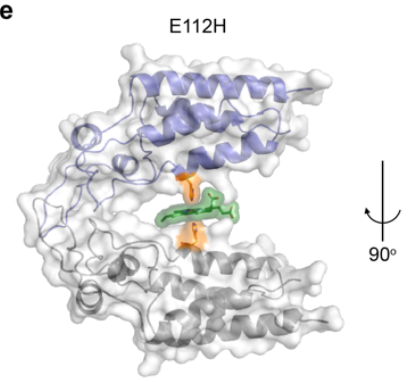

g

\begin{tabular}{cc} 
Position & $\begin{array}{c}\mathrm{N}^{\varepsilon}-\text { Fe distance } \\
\text { after minimization }\end{array}$ \\
\hline 101 & $2.4 \AA$ \\
105 & $2.3 \AA$ \\
108 & $2.5 \AA$ \\
109 & $2.3 \AA$ \\
112 & $2.4 \AA$ \\
116 & $2.2 \AA$
\end{tabular}

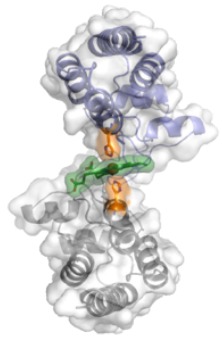

b
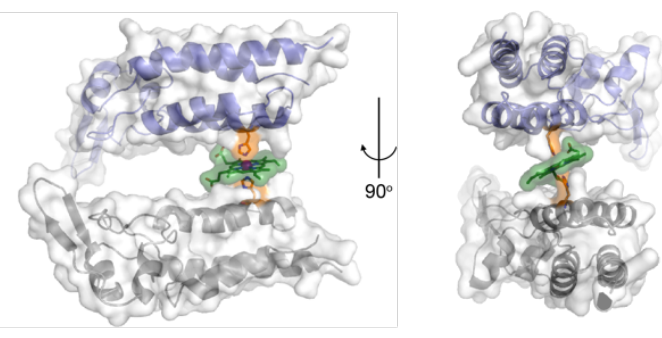

d

Q109H
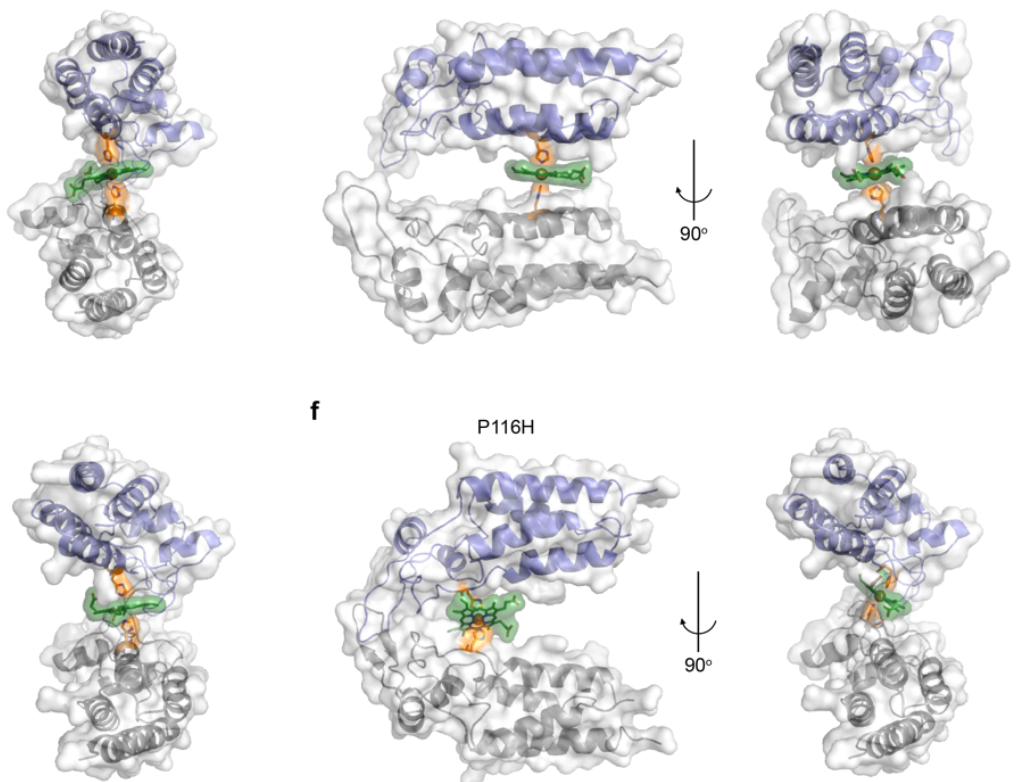

f
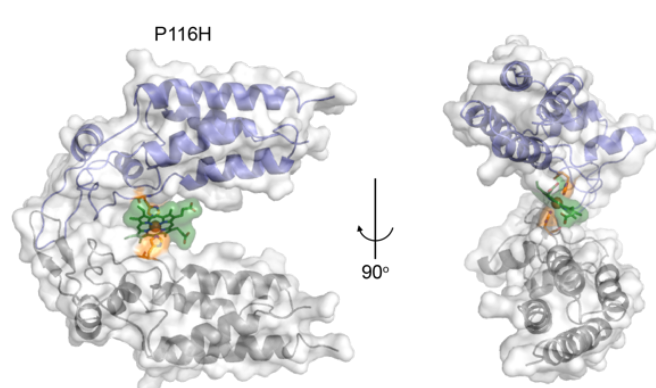

h
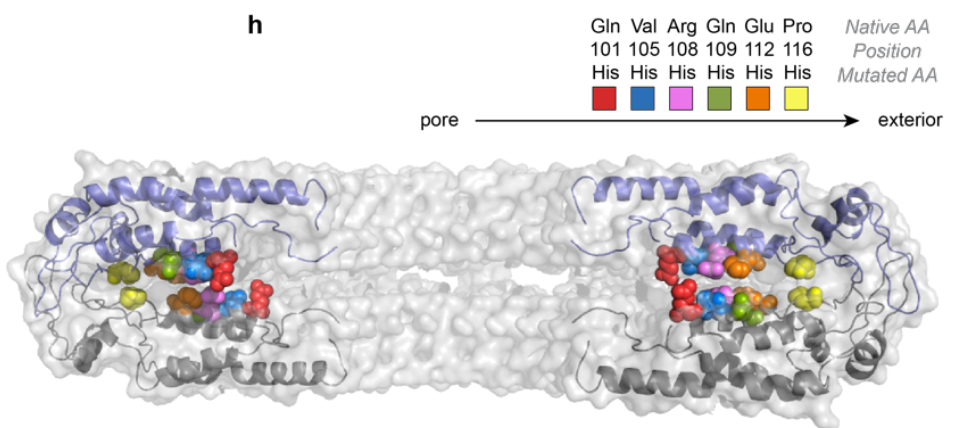

Figure S2. Computationally modeled heme binding pockets of six cpTMV mutants. A single top-bottom pair with the heme binding to the introduced histidine residues is shown for each mutant as: (a) Q101H, (b) V105, (c) $\mathrm{R} 108 \mathrm{H}$, (d) Q109H, (e) E112H, (f) P116H. (g) The $\mathrm{N}^{\varepsilon}-$ Fe distances after computational minimization are listed. (h) The spatial distribution of six possible mutation sites is shown within the double-disk assembly. 


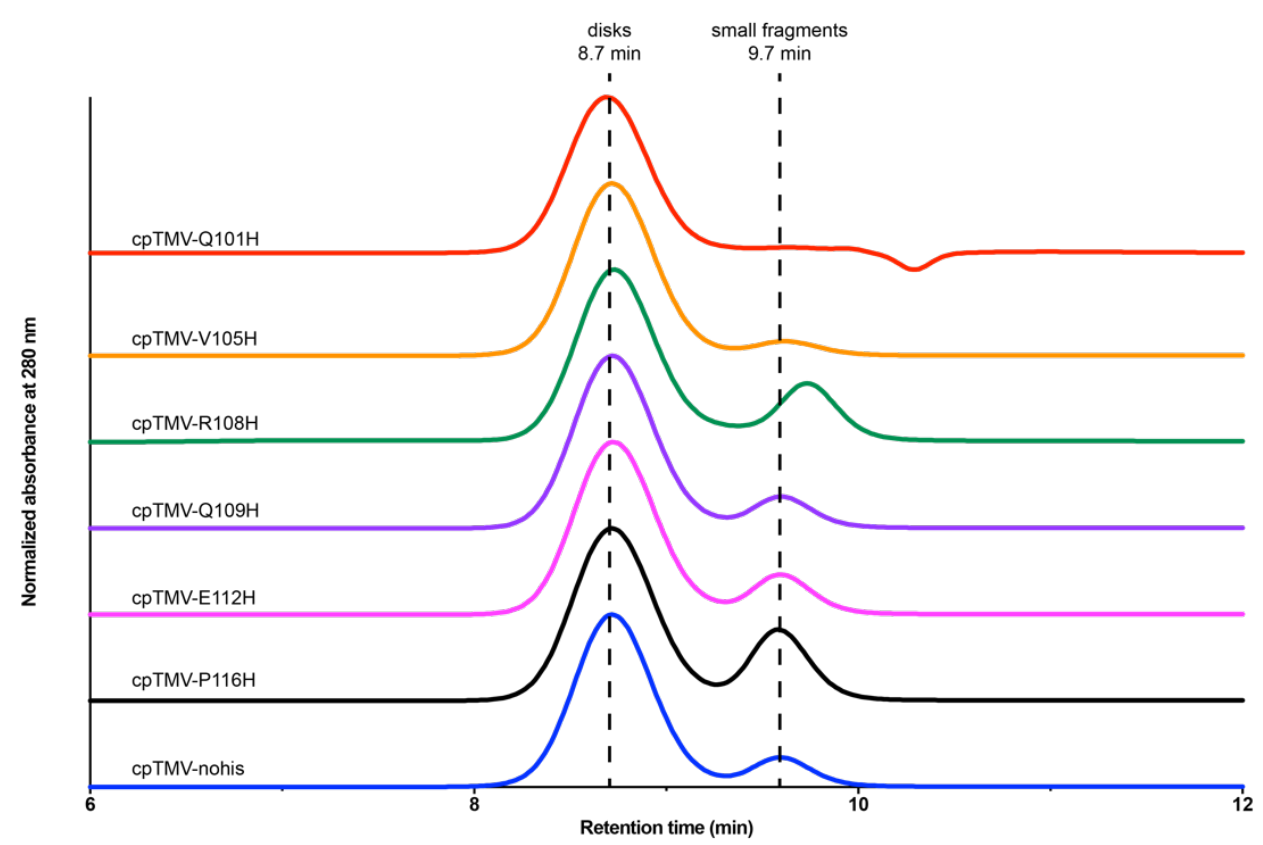

Figure S3. Assembly of cpTMV mutants characterized by size exclusion chromatography (SEC). All six mutants form mainly double-disk assemblies (eluting at around $8.7 \mathrm{~min}$ ) mixed with different ratios of smaller fragments (eluting at around $9.7 \mathrm{~min}$ ). The SEC traces are colored differently as Q101H (red), V105H (orange), R108H (green), Q109H (purple), E112H (pink), P116H (black), and cpTMV-nohis (blue). 


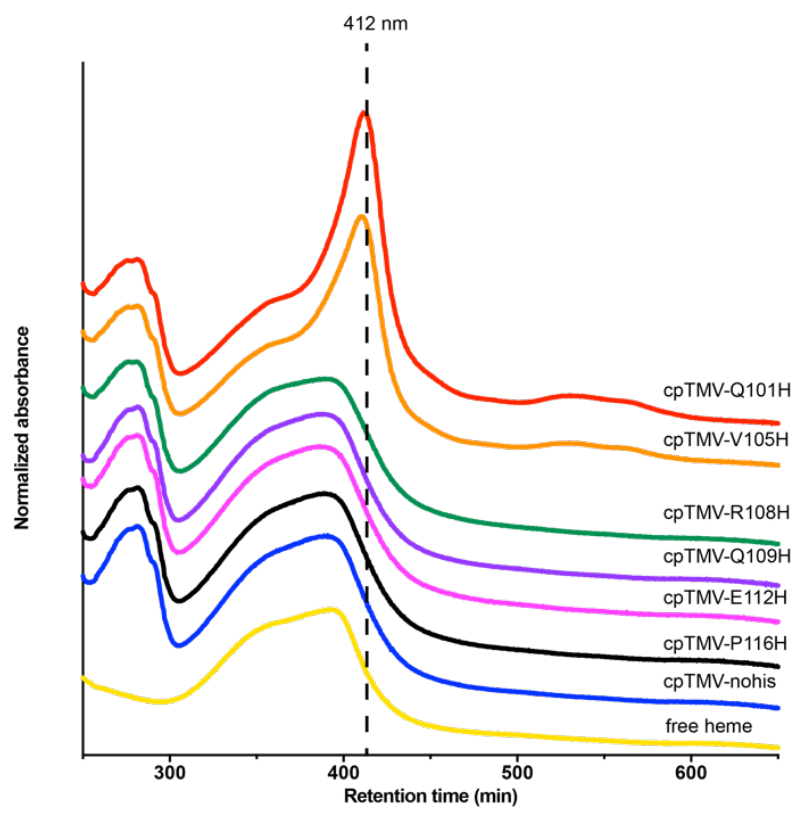

b

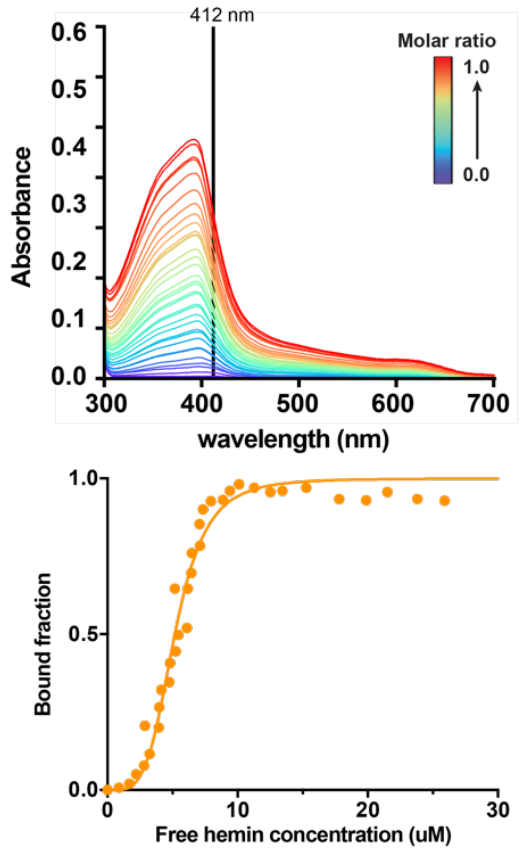

Figure S4. Characterization of heme binding events of cpTMV mutants. All six mutants were incubated at the same concentration $(20 \mu \mathrm{M})$ with 0.5 equiv. heme, and the UV-Vis spectra were recorded. Free heme in buffer (yellow) and heme incubated with non-histidine containing cpTMV (cpTMV-nohis, blue) are included for comparison. (a) UV-vis spectra suggested the heme binding ability of two mutants Q101H (red) and $\mathrm{V} 105 \mathrm{H}$ (orange), as evidenced by the emergence of peaks at 412, 530, and $564 \mathrm{~nm}$. The same spectral profile as two controls (free heme and cpTMV-nohis) was observed in the rest of the mutants R108H (green), Q109H (purple), E112H (pink) and $\mathrm{P} 116 \mathrm{H}$ (black). (b) The same set of titration experiments was conducted for cpTMV-nohis in parallel to cpTMV-Q101H. The UV-vis spectra of varied concentrations of heme incubated with cpTMV-nohis are shown. The only change observed for cpTMV-nohis is the proportional increase of the absorbance as the heme concentration increased. (c) The concentration of free heme and the bound fraction was calculated for each titration data point to give the Hill coefficient as 4.26 and the apparent dissociation constant as $5.18 \mu \mathrm{M}$ by fitting with the Hill-Langmuir equation. ${ }^{2}$ 
a

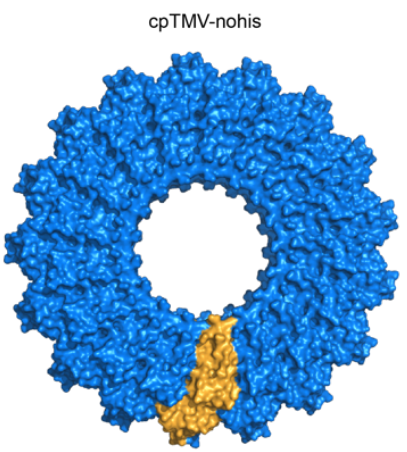

d

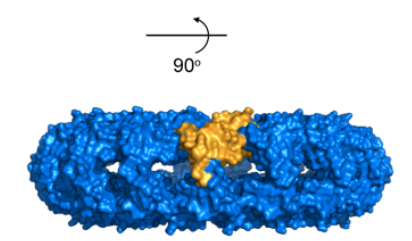

g

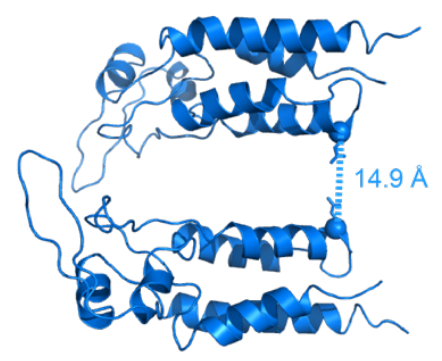

b

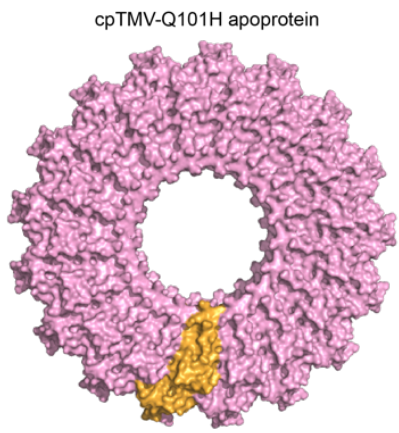

e
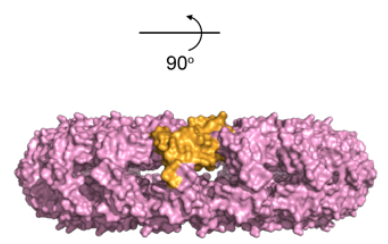

h

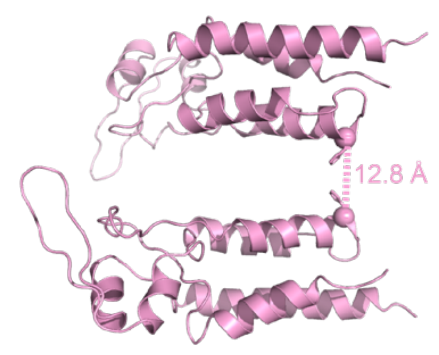

c

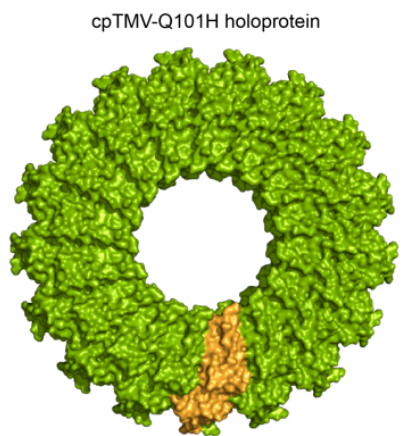

f
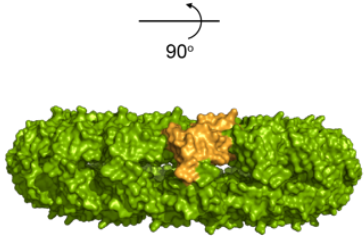

i

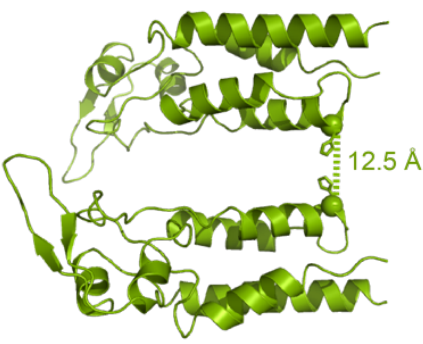

Figure S5. Structural comparisons for three cpTMV structures. The mutation and heme binding did not alter the assembly symmetry and overall folding pattern of cpTMV relative to the previously published crystal structure (cpTMV-nohis, PDB code: 3KML). The top-down views of the double-disk assembly are shown for (a) cpTMV-nohis (blue), (b) cpTMV-Q101H apoprotein (pink), and (c) cpTMV-Q101H holoprotein (green), with one monomer highlighted in orange. The side views of the assembly are shown as well for (d) cpTMV-nohis (blue), (e) cpTMV-Q101H apoprotein (pink), and (f) cpTMV-Q101H holoprotein (green). The distance between analogous $\mathrm{C}_{\alpha}$ atoms in the top monomer and the bottom monomer were calculated for the 101 site as (g) $14.9 \AA$ in cpTMV-nohis (blue), (h) $12.8 \AA$ in cpTMV-Q101H apoprotein (pink), and (i) $12.5 \AA$ in cpTMV-Q101H holoprotein (green). 
a

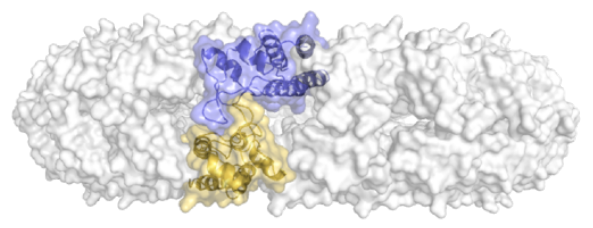

b

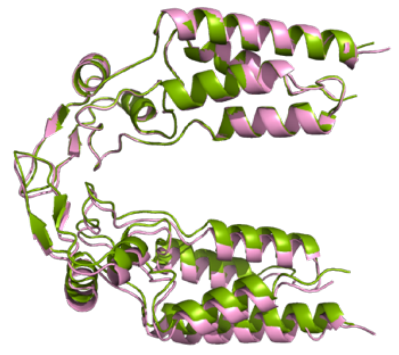

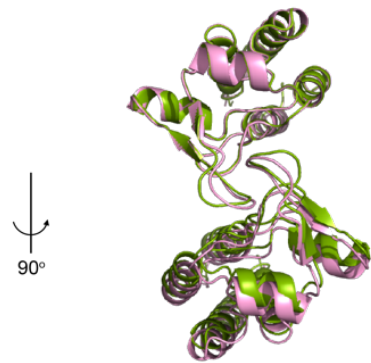

c
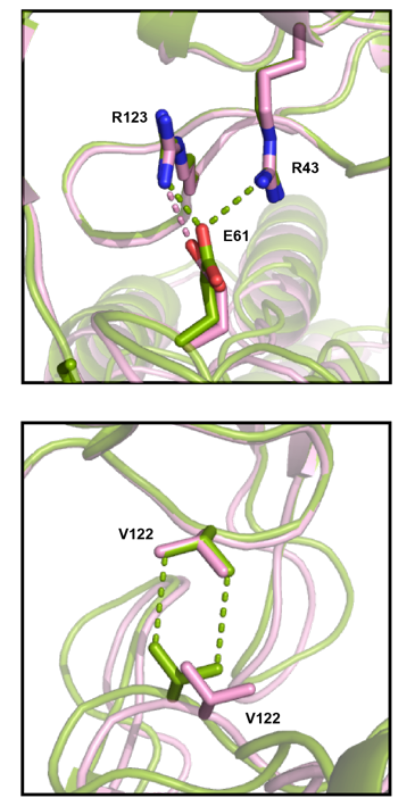

d

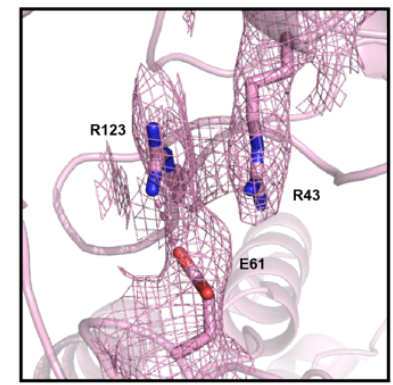

g

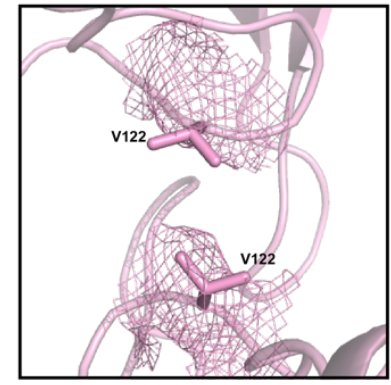

e

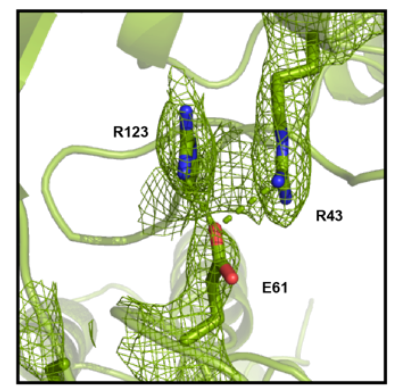

h

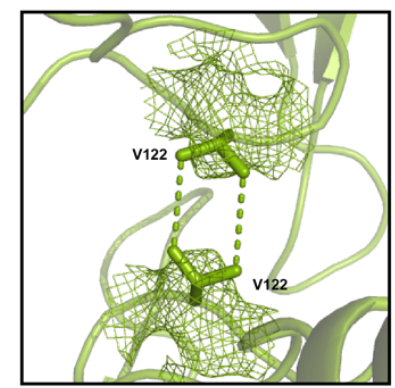

Figure S6. Analysis of the protein-protein interaction changes upon heme binding. As shown in (a), two interacting monomers sitting in top and bottom disks separately are highlighted as purple and yellow. (b) Two such interacting pairs from cpTMV-Q101H apoprotein (pink) and holoprotein (green), separately, are extracted and aligned. With the top monomers superimposed, the distances to the bottom monomers is clearly reduced after the introduction of the metal cofactor. The peripheral loop regions (residue 53-66 and 117-125) are critical to holding the top and bottom disks together and were thought to exert the main force to accommodate the global change propagated throughout the whole disk assembly upon heme binding. Although the electron density in the loop regions was generally of poorer quality, specific side chain interactions that change upon heme coordination are suggested. (c) In cpTMV-Q101H holoprotein (green), E61 in the bottom monomer forms salt bridges with both R123 and R43 in the top monomer. In the cpTMV-Q101H apoprotein (pink), E61 is further away from the two arginines and forms weaker electrostatic interaction with them. The $2 \mathrm{mF}_{\mathrm{O}}-\mathrm{DF}_{\mathrm{C}}$ map is contoured at the $1.2 \sigma$ level around the three residues mentioned above for (d) cpTMV-Q101H apoprotein (pink meshed line) and (e) cpTMV-Q101H holoprotein (green meshed line). (f) Stronger hydrophobic interactions were observed between V122 of the top and bottom monomers in cpTMV-Q101H holoprotein. The $2 \mathrm{mF}_{\mathrm{O}}-\mathrm{DF}_{\mathrm{C}}$ map is contoured at the $1.2 \sigma$ level around the two residues mentioned above for (g) cpTMV-Q101H apoprotein (pink meshed line) and (h) cpTMV-Q101H holoprotein (green meshed line). There might be additional interactions present to contribute to the assembly rearrangement; however, due to the poor electron density of the loop region, they can not be identified. 
a
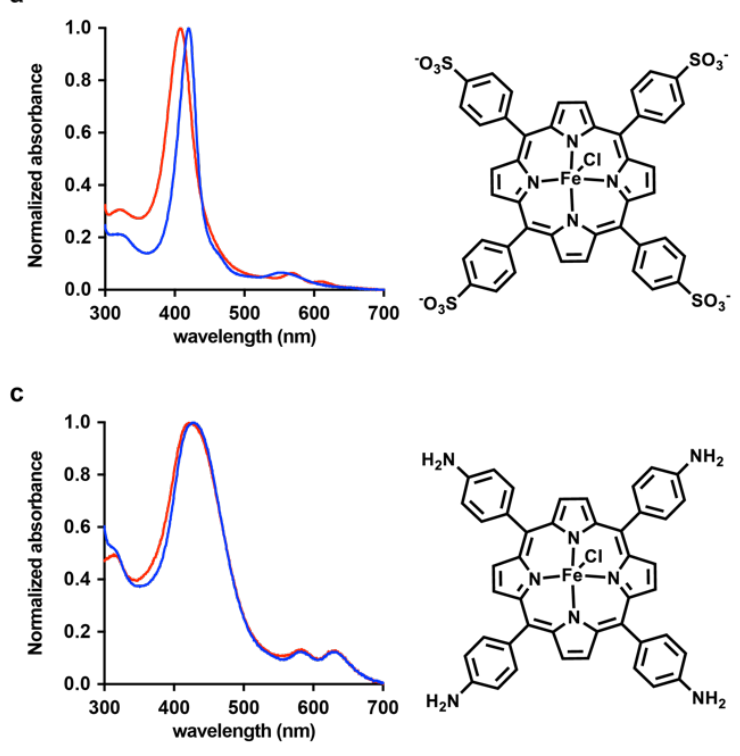

e

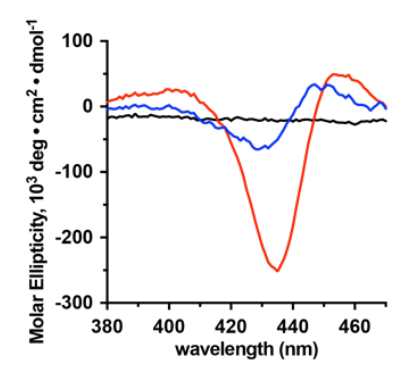

b

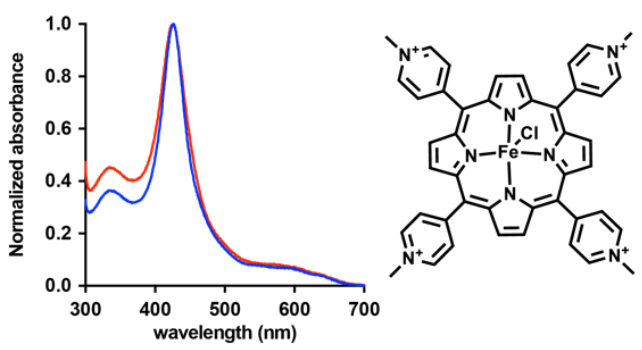

d

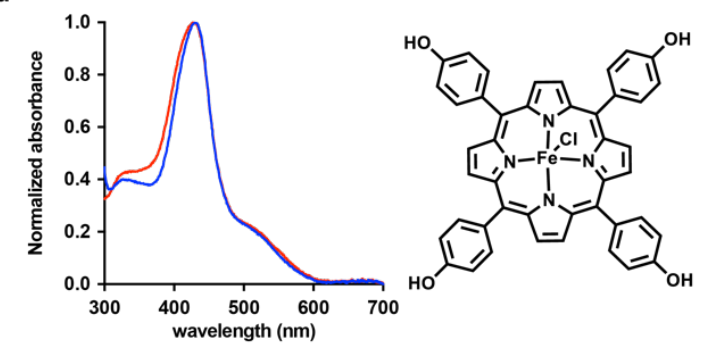

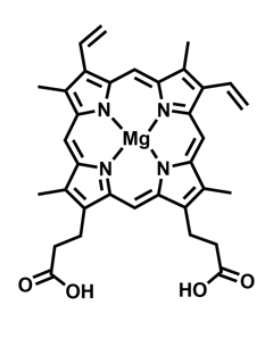

Figure S7. Characterization of cpTMV-Q101H binding to other types of metal porphyrins. The UV-vis spectra of iron porphyrins with varied side chains were measured after incubation with either the cpTMV-Q101H (red line) or cpTMV-nohis (blue line, control). A shift in both Soret band and Q band was expected for successful binding and was only observed for porphyrins with (a) sulfonatophenyl side groups. No difference in spectra comparing the data of cpTMV-Q101H and cpTMV-nohis for the porphyrins with (b) $N$-methyl-4-pyridylside groups, (c) aminophenyl side groups, and (d) hydroxyphenyl side groups. In (e) the $\mathrm{CD}$ spectra are shown for $\mathrm{Mg}$ (II) protoporphyrin IX (MgPPIX) alone in solution (black line), incubated with cpTMV-nohis (blue line), and with cpTMV-Q101H (red line). Unlike the free MgPPIX (black line) which did not display any signal in CD, MgPPIX incubated with cpTMV-nohis (blue line) displayed a CD signal that is likely due to the presence of non-specific interactions. The much more obvious Cotton effect was observed for cpTMV-Q101H data suggesting that additional interactions were present explicitly for cpTMV-Q101H, which is very likely due to the metal coordination. 


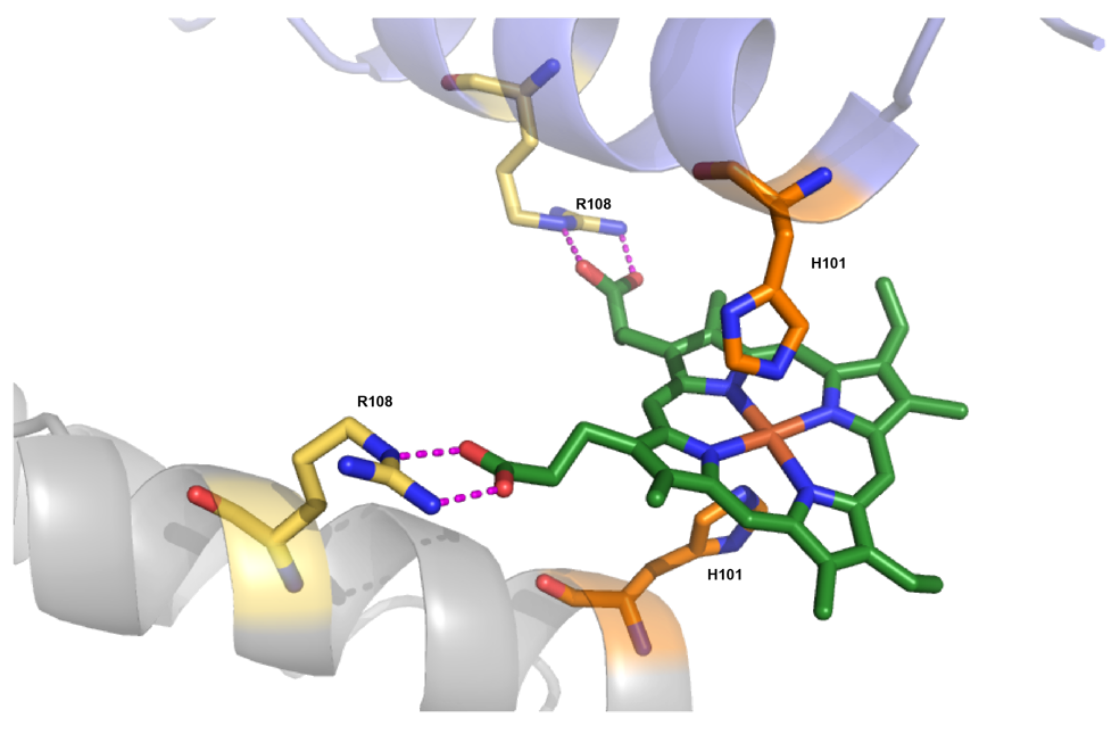

Figure S8. Modeled interaction between the heme and protein side chains. Molecular Operating Environment $(\mathrm{MOE})^{3}$ was used to model the possible interactions between the heme and the protein.The propionate groups of heme and the surrounding protein side chains were set to move freely. The porphyrin core, $\mathrm{H} 101$ and the surrounding residues (within $10 \AA$ ) were set contrained and the rest of the protein was fixed (frozen). One possible conformation is shown that possesses hydrogen bonding (purple dashed line) between the propionate groups and R108 residues.

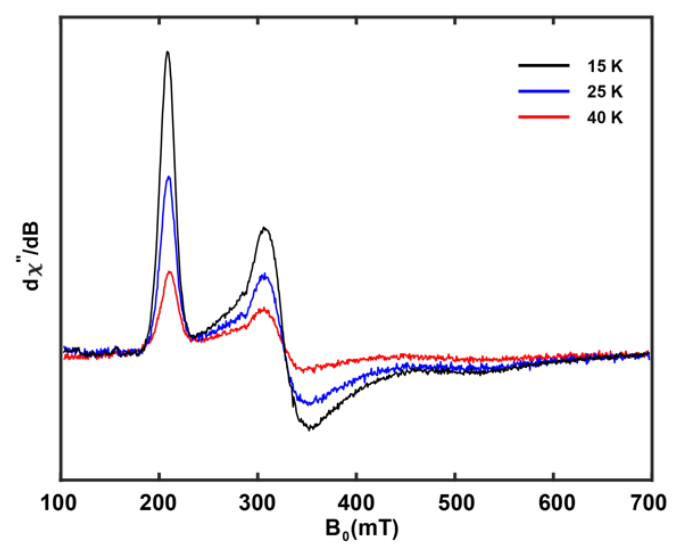

Figure S9. Temperature dependent EPR spectra. X-band EPR spectra of hemes bound to cpTMV-Q101H in their resting ferric states were recorded at $15 \mathrm{~K}$ (black line), $25 \mathrm{~K}$ (blue line) and $40 \mathrm{~K}$ (red line). The spectral features were retained with an intensity decrease of the peaks as temperature increased. Experimental parameters: temperature $=15 \mathrm{~K}$; microwave frequency $=9.38 \mathrm{GHz}$; microwave power $=2 \mathrm{~mW}$ (no saturation); conversion time $=100 \mathrm{~ms}$; modulation amplitude $=0.8 \mathrm{mT}$; and modulation frequency $=100$ kHz. 
a

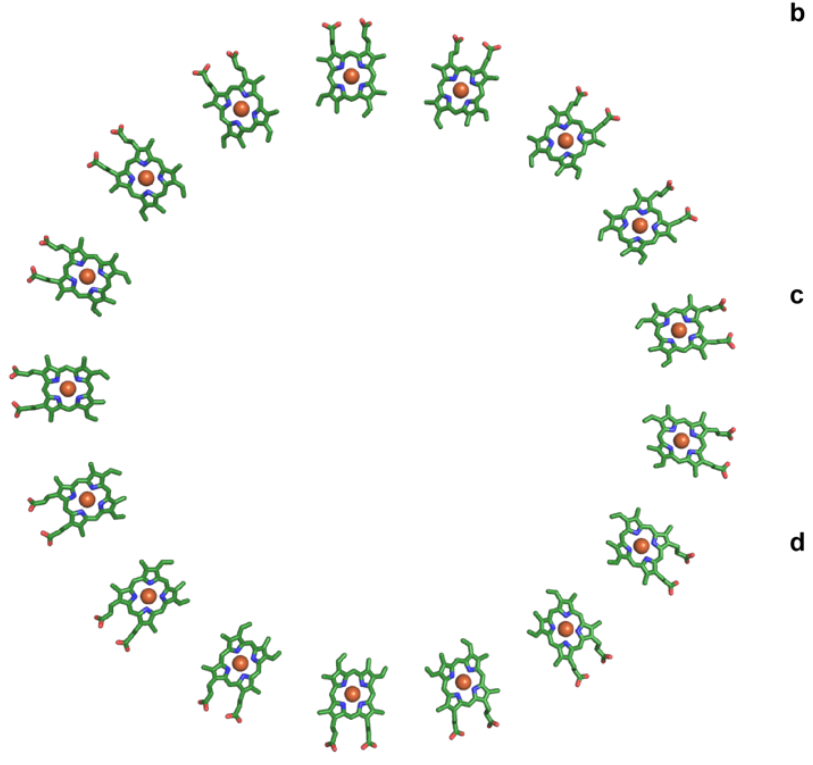

b

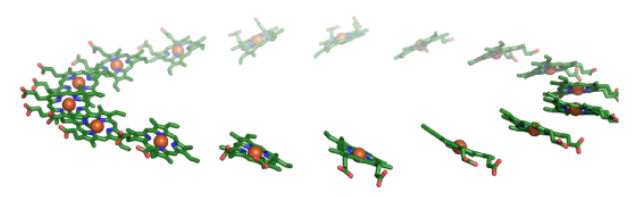

C
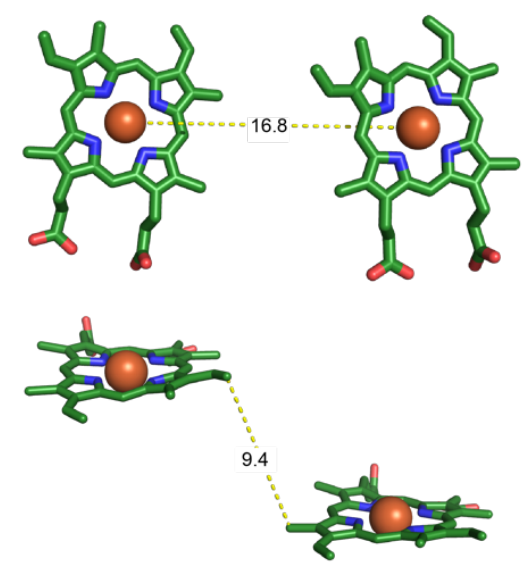

Figure S10. Arrangement of heme arrays templated by cpTMV. The full array of 17 hemes forming a ring is shown as a top-down view in (a) and a side view in (b). Two adjacent hemes were isolated to show the relative orientation between each other as a top-down view in (c) and a side view in (d). The iron-to-iron distance was measured as $16.8 \AA$ and the edge-to-edge distance was measured as $9.4 \AA$. 


\section{S4 References}

(1) Dedeo, M. T.; Duderstadt, K. E.; Berger, J. M.; Francis, M. B. Nanoscale Protein Assemblies from a Circular Permutant of the Tobacco Mosaic Virus. Nano Lett. 2010, 10 (1), 181-186.

(2) Weiss, J. N. The Hill Equation Revisited: Uses and Misuses. FASEB J. 1997, 11 (11), 835-841.

(3) Molecular Operating Environment (MOE), 2019.02; Chemical Computing Group ULC: Montreal, QC, Canada, 2019. 